\title{
Characterization of the ovine ribosomal protein SA gene and its pseudogenes
}

\author{
Alice Van den Broeke', Mario Van Poucke', Ane Marcos-Carcavilla² ${ }^{1}$ Karine Hugot ${ }^{3}$, Hélène Hayes ${ }^{3}$, Maud Bertaud ${ }^{3}$,
} Alex Van Zeveren ${ }^{1}$, Luc J Peelman ${ }^{1 *}$

\begin{abstract}
Background: The ribosomal protein SA (RPSA), previously named 37-kDa laminin receptor precursor/67-kDa laminin receptor (LRP/LR) is a multifunctional protein that plays a role in a number of pathological processes, such as cancer and prion diseases. In all investigated species, RPSA is a member of a multicopy gene family consisting of one full length functional gene and several pseudogenes. Therefore, for studies on RPSA related pathways/ pathologies, it is important to characterize the whole family and to address the possible function of the other RPSA family members. The present work aims at deciphering the RPSA family in sheep.

Results: In addition to the full length functional ovine RPSA gene, 11 other members of this multicopy gene family, all processed pseudogenes, were identified. Comparison between the RPSA transcript and these pseudogenes shows a large variety in sequence identities ranging from $99 \%$ to $74 \%$. Only one of the 11 pseudogenes, i.e. RPSAP7, shares the same open reading frame (ORF) of 295 amino acids with the RPSA gene, differing in only one amino acid. All members of the RPSA family were annotated by comparative mapping and fluorescence in situ hybridization (FISH) localization. Transcription was investigated in the cerebrum, cerebellum, spleen, muscle, lymph node, duodenum and blood, and transcripts were detected for 6 of the 11 pseudogenes in some of these tissues.

Conclusions: In the present work we have characterized the ovine RPSA family. Our results have revealed the existence of 11 ovine RPSA pseudogenes and provide new data on their structure and sequence. Such information will facilitate molecular studies of the functional RPSA gene taking into account the existence of these pseudogenes in the design of experiments. It remains to be investigated if the transcribed members are functional as regulatory non-coding RNA or as functional proteins.
\end{abstract}

\section{Background}

The ribosomal protein SA (RPSA), previously named 37-kDa laminin receptor precursor/67-kDa laminin receptor (LRP/LR) is a multifunctional protein. In the nucleus it binds to DNA via the histones $\mathrm{H} 2 \mathrm{~A}, \mathrm{H} 2 \mathrm{~B}$ and $\mathrm{H} 4$ [1], in the cytoplasm it is associated with the $40 \mathrm{~S}$ ribosomal subunit [2], and at the cell surface it acts as a receptor for a number of components i.e. laminin, elastin, the green tea catechin epigallocatechin-3-gallate (EGCG), carbohydrates, the prion protein, different viruses like Dengue virus, Sindbis virus, Venezuelean Equine Encephalitis virus and Adeno-associated-viruses

\footnotetext{
* Correspondence: Luc.Peelman@UGent.be

'Department of Nutrition, Genetics and Ethology, Faculty of Veterinary Medicine, Ghent University, Heidestraat 19, B-9820 Merelbeke, Belgium
}

and various bacteria like Streptococcus pneumoniae, Neisseria meningitidis and Haemophilus influenza [2,3].

The receptor is involved in many pathological processes. It is upregulated in cancer and its expression is positively correlated with metastasis and the aggressiveness of tumour cells in breast, ovary, lung, prostate and cervical carcinomas [2]. In the context of prion disease, RPSA is needed for the internalization and propagation of prion proteins [2]. Several therapeutic approaches based on down-regulation (e.g. via RNA interference) and/or blocking (e.g. with specific antibodies or transdominant negative mutants) of the receptor result in reduced adhesion, migration and invasion of tumour cells [4-7], and reduced accumulation of the pathogenic isoform of the prion protein in many organs involved in the pathogenesis of transmissible spongiform
C Biomed Central

() 2010 Van den Broeke et al; licensee BioMed Central Ltd. This is an Open Access article distributed under the terms of the Creative Commons Attribution License (http://creativecommons.org/licenses/by/2.0), which permits unrestricted use, distribution, and reproduction in any medium, provided the original work is properly cited. 
encephalopathies [8-12], leading to a significant prolongation of the pre-clinical phase or survival time after the occurrence of the first symptoms [10-12].

In addition, it has been shown that binding of green tea catechin EGCG to RPSA causes anti-thrombotic, anti-allergic and anti-obesity effects and mediates cancer prevention by inhibiting cell growth [13-16], thus RPSA is a target in new therapies against this large group of diseases.

However, in order to unravel the multiple pathways in which RPSA is involved and to develop RPSA-based diagnostic/therapeutic tools, it is necessary first to characterize in full detail the complex genetic background of RPSA. Indeed, previous studies have shown that in most investigated species thus far, RPSA is a member of a multicopy gene family consisting of one full length functional gene and several pseudogenes (e.g. at least 63 in man; Table 1). Moreover, the presence of pseudogenes in a genome can interfere with molecular studies of the corresponding functional gene (i.e. sequencing, mapping, polymorphism detection, genotyping, association analysis, mRNA expression studies, ...) and transcribed pseudogenes can produce endogenous small interfering RNAs that regulate the expression of the functional gene or other genes [17].

Previously, Marcos-Carcavilla et al. [18] have postulated the existence of an ovine RPSA pseudogene. The present work aims at providing a genetic basis for future studies on RPSA related pathways/pathologies in sheep by identifying and characterizing the complex RPSA gene family.

\section{Results and Discussion}

BAC screening and STS content mapping

Eight different primer pairs were designed in conserved ovine RPSA regions identified by aligning previously described mRNA and expressed sequence tag (EST) sequences, representing each exon at least once. Using these primers, 34 bacterial artificial chromosome (BAC) clones, containing members of the RPSA family, were isolated by PCR screening of the INRA sheep BAC library [19], with an annealing temperature $(\mathrm{Ta})$ that was at least $8^{\circ} \mathrm{C}$ lower than the melting temperature (Tm) of the primers to allow primer mismatches (Additional file 1). By sequence tagged site (STS) content mapping, performed with 54 unique STS primer pairs that were designed from the $68 \mathrm{BAC}$ end sequences (BES) [GenBank:GS375851-GS375918], 6 mini-contigs could be constructed and another 6 single BAC clones could be identified, each containing a different family member of the ovine RPSA family (Figure 1 and 2; Additional file 2).

\section{Characterization of the 12 RPSA gene family members}

Each member of the RPSA gene family was sequenced by direct sequencing on BAC DNA starting with the PCR primers as sequencing primers and finishing by primer walking. The sequences were assembled with the CAP3 program [20] and annotated with BLAST [21].

The full length functional gene, that was first described by Marcos-Carcavilla et al. [18], was present in one of the contigs composed of 6 BAC clones [Genbank:GQ202529]. We have sequenced for the first time, the complete intron 3, comprising $8846 \mathrm{bp}$, which like the other introns, has consensus acceptor and donor splice sites. The full length ovine RPSA gene consist thus of $13287 \mathrm{bp}$.

Besides the full length functional gene, 11 other RPSA gene family members were sequenced [GenBank: GQ202530-GQ202540]. A schematic representation of all the family members, based on sequence alignments with the full length functional gene (Additional file 3), is included in Figure 3. They all are considered as processed pseudogenes and in accordance with RPSA pseudogenes described in other species, they have been assigned the names RPSAP1-RPSAP11. Pseudogenes

Table 1 Number of RPSA pseudogenes in different species identified so far

\begin{tabular}{|c|c|c|c|}
\hline Species & Processed pseudogenes/transcribed & Duplicated pseudogenes & Reference \\
\hline Homo sapiens & $63^{(a)} / 1^{(b)}$ & / & $\begin{array}{l}\text { Balasubramanian et al. (2009) [45] } \\
\text { Asano et al. (2004)[46] }\end{array}$ \\
\hline Bos taurus & $60^{(c)} / 1^{(b)}$ & / & Germerodt et al. (2004) [32] $]^{(b)}$ \\
\hline Sus scrofa & $2^{(b)}$ & $1^{(b)}$ & Knorr et al. (2007) [47] $]^{(\mathrm{b})}$ \\
\hline Mus musculus & $45^{(\mathrm{a})} / 2^{(\mathrm{b})}$ & / & $\begin{array}{l}\text { Balasubramanian et al. (2009) [45] }]^{(a)} \\
\text { Fernandez et al. (1991) [48] }\end{array}$ \\
\hline Gallus gallus & / & / & Bignon et al. (1991) [49] ${ }^{(b)}$ \\
\hline Ovis aries & / & $1^{(\mathrm{b})} ?$ & Marcos-Carcavilla et al. (2008)[18] $]^{(b)}$ \\
\hline Pan troglodytes & $52^{(a)}$ & & Balasubramanian et al. (2009) [45] ${ }^{(a)}$ \\
\hline Rattus norvegicus & $45^{(\mathrm{a})}$ & & Balasubramanian et al. (2009)[45] $]^{(a)}$ \\
\hline
\end{tabular}




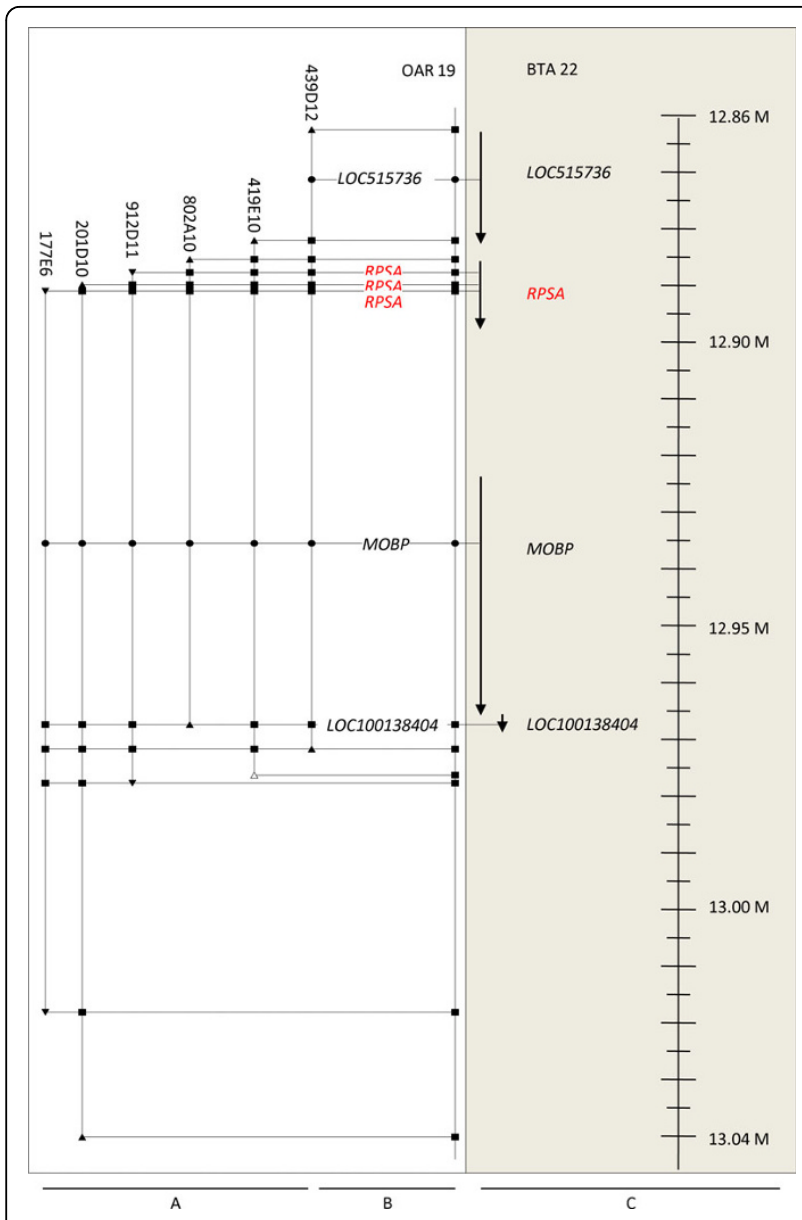

Figure 1 Comparative mapping of the region of RPSA in sheep and cattle. The ovine BAC mini-contig is drawn in part A. Triangles represent $B A C$ end sequences; pointing towards the 3 '-end of the BAC clone. Black triangles represent BES from which primers were designed to construct the mini-contig. White triangles are BES from which it was impossible to design STS-primers. Black squares show overlaps between BES and other BAC clones. Black circles represent genes annotated by PCR. Annotated sequences are shown in a plane map in part B. The position and orientation of the genes present in the syntenic region of Bos taurus are represented with arrows (C).

arise in 2 different manners: either by retrotransposition of the mRNA of the ancestral gene into the genome or by duplication of genomic DNA [22]. The first class is known as processed pseudogenes, the second one as non processed pseudogenes. The majority of the pseudogenes are processed and originate from housekeeping genes, with ribosomal protein genes as largest subgroup $[22,23]$. As processed pseudogenes are inserted without internal promoter, they are released from selection pressure and accumulate mutations during evolution leading to frameshift mutations and/or premature stopcodons which prevents them of encoding a functional protein
[24]. In some cases nevertheless, they have obtained a (regulatory) function [17].

To investigate this possibility, all the RPSA pseudogenes were further characterized in silico and their main characteristics are listed in detail in Table 2 and 3. Comparison with the full length RPSA gene transcript shows that the pseudogenes vary greatly both in structure and sequence identity. These differences range from structurally identical pseudogenes sharing $99 \%$ sequence identity (RPSAP7) to pseudogenes lacking half of the gene (RPSAP8, RPSAP9 and RPSAP10) or containing many deletions throughout the whole gene sharing a sequence identity of only $74 \%$ (RPSAP2).

Analysis of the primer binding sites in the pseudogenes showed that in our experimental design the screening primers could anneal to targets down to $83 \%$ sequence identity, even in the case of RPSAP2.

All BAC clones and thus all RPSA family members were isolated with at least 2 primer pairs and there was no concordance between the number of BACs in a mini-contig and the level of sequence identity with RPSA. We conclude that it is most likely that we have isolated all the ovine RPSA pseudogenes sharing a high level of sequence identity and that therefore can interfere with the functional RPSA gene in genetic studies.

To obtain a first indication of possible functionality, in silico ORF and promoter prediction analysis were carried out.

The pseudogene RPSAP7 is the only member sharing almost an identical ORF with the full length RPSA gene. The only one amino acid difference (amino acid 31: $\mathrm{D} \rightarrow \mathrm{G}$ ) is located in the intracellular part of the receptor that does not belong to any binding site. All the other pseudogenes either lack the start codon or contain a premature stop codon due to nonsense or frameshift mutations. The size of the potential ORF of the other pseudogenes varies and the largest reaches 171 amino acids sharing $100 \%$ identity with RPSA (Table 2). Most ORFs lie in the intracellular region of RPSA (amino acid 1-101). In case of RPSAP6, RPSAP8, RPSAP10 and RPSAP11, the ORF contains a part of the binding sites of RPSA with PrP (direct binding aa 161-180; indirect binding aa 180-285 [25]), but most of them have a low level of amino acid identity.

In silico promoter analysis predicted a possible promoter for RPSAP1, RPSAP2, RPSAP4, RPSAP8, RPSAP9 and RPSAP10 (Additional file 4). A consensus polyadenylation signal is present in 7 of the 11 pseudogenes (including RPSAP7).

Repeated sequences were identified with Repeatmasker [26] and showed that 4 pseudogenes are disrupted by interspersed repeats belonging to the class/family SINE/ RTE-BovB, SINE/BovA, tRNA and SINE/tRNA-Glu, and that 7 pseudogenes were flanked by repeats belonging to 


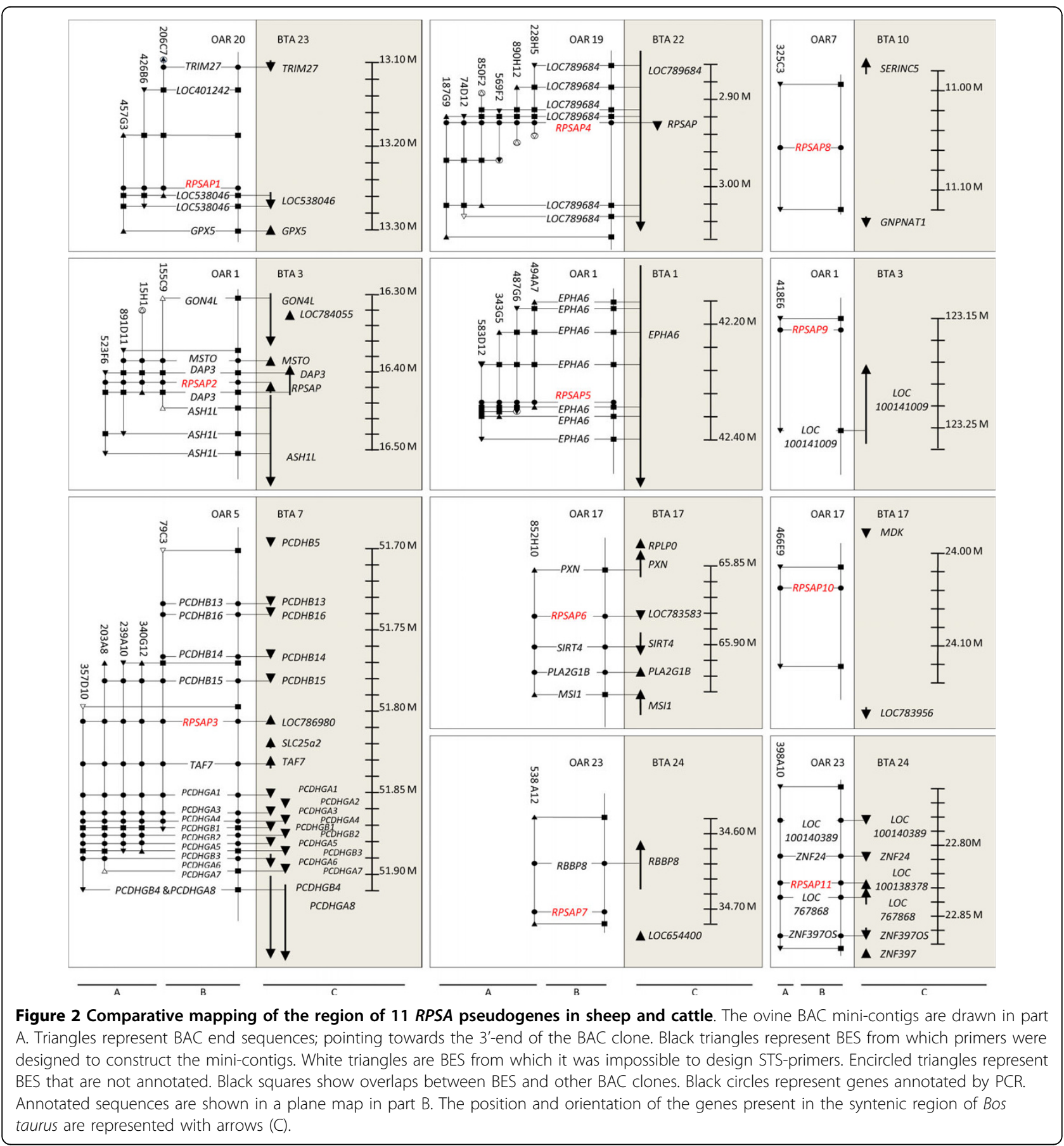

the SINE, LINE, tRNA, LTR and simple repeat classes. According to Zhang et al. [27], processed pseudogenes are mostly found in genomic regions with a relatively low GC content, as do LINE repeats. Thus, it is not surprising that such repeats are present in the regions flanking many of the RPSA pseudogenes.

A remarkable observation is that part of the RPSA intron 4 , containing the small nucleolar RNA (snoRNA) SNORA62, is present in the RPSAP8 and RPSAP9 pseudogenes. Therefore, these pseudogenes can be considered as semi-processed pseudogenes, which are very rarely reported and defined by Zhang et al. as "pseudogenes that contain remnant introns, which suggests that they were derived from semi-processed RNA transcripts" [28].

SnoRNAs are encoded in introns of ribosomal protein genes and other housekeeping genes [29,30], and are responsible for both sequence-specific methylation and pseudouridilation of RNA [31]. SNORA62 isan H/ACA 


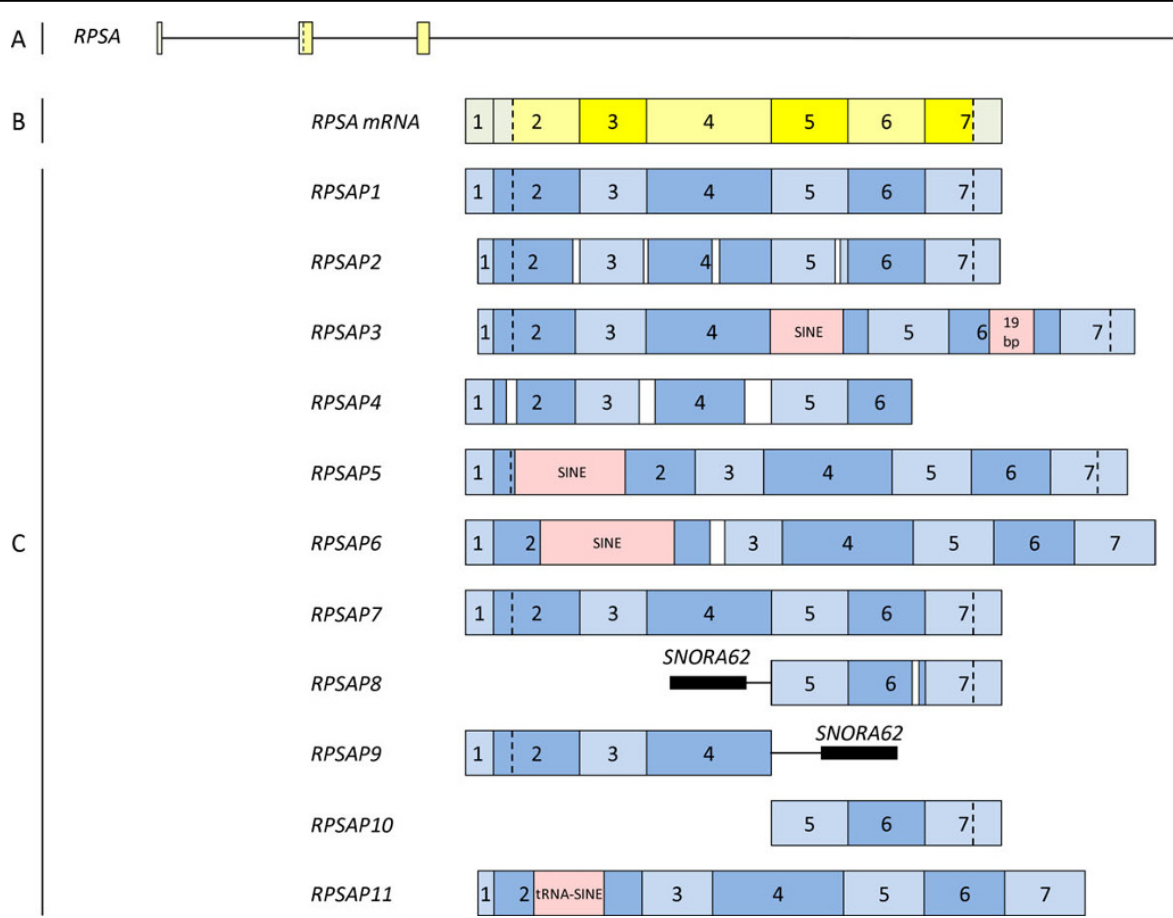

Figure 3 Schematic overview of the RPSA (pseudo)genes. The genomic structure of the ovine RPSA gene is drawn in part $\mathrm{A}$, the mRNA of the RPSA gene is drawn in part B. Part C represents the genomic structure of the different RPSA pseudogenes. The squares represent exons and the lines stand for introns. The coding sequence (CDS) is drawn in yellow; the untranslated sequences in green. The blue squares are parts of the pseudogene sequence that are analogous with the exons of the RPSA mRNA. The pink squares symbolize interspersed sequences and the white gaps deletions. SNORA62 is represented as a black square. Start codons and stop codons, analogous with the ones of RPSA, are represented by a dotted line.

box snoRNA that guides the isomerization of uridine into pseudouridine [30] by binding with 2 uridines of 28S rRNA (U3830 and U3832). Sequence comparison shows that these important regions display mutations in RPSAP8 but are conserved in RPSAP9 (Figure 4). As a result, the paralog of SNORA62 is probably not functional in RPSAP8. In RPSAP9 on the other hand, the paralog of SNORA62 could, in addition to SNORA62, exhibit the function of pseudouridilation in case of transcription [29]. Marcos-Carcavilla et al. [18] had already postulated the existence of a non-processed pseudogene that differed from the active RPSA gene by the absence of a $\mathrm{G}$ at position 29 of intron 4. Thus, we hypothesize that this previously mentioned non-processed pseudogene is in fact the semi-processed pseudogene RPSAP9, because it lacks the $\mathrm{G}$ at position 29 of intron 4 and it can co-amplify with the active RPSA gene because of its high sequence identity (98\%).

\section{Annotation of the mini-contigs by comparative mapping and FISH localization}

The genomic regions containing the 12 members of the RPSA gene family were further investigated by sequence comparison of both BES and internal BAC sequences using NCBI BLAST [21] (Figure 1 and 2). Sixty-two of the $68 \mathrm{BES}$ were annotated while the remaining 6 contained either too many repeat sequences or no specific orthologous sequence to allow annotation. The different characteristics (length, repeat sequences and genes) are listed in the Additional file 5. Based on sequence annotation results, 40 ovine genes, of which 37 have not been described in sheep yet, could be mapped on the mini-contigs by comparative mapping with the bovine genome (Figure 1 and 2). The primers used to perform the PCR for annotating the genes, together with another 18 optimized primer pairs, amplifying genes not present in the mini-contig but flanking the genomic region of the different RPSA family members, are listed in Additional file 6 .

The 11 pseudogenes were localized by FISH on different sheep chromosomes (see Table 4 and pictures of the FISH experiments in Additional file 7). All the localizations confirmed the positions predicted from the genes present in the mini-contigs by using the online tool Virtual Sheep Genome Assembly v2.0.

As expected, most RPSA pseudogenes are located in intergenic regions except 3 found in the intron of other genes (RPSAP2 in DAP3; RPSAP4 in LOC789684 and 
Table 2 Characteristics of RPSA (pseudo)genes-general characteristics

\begin{tabular}{|c|c|c|c|c|c|c|c|}
\hline Gene & $\begin{array}{l}\text { Acc. No. } \\
\text { GenBank }\end{array}$ & $\begin{array}{l}\% \text { nucleotide } \\
\text { identity } R P S A\end{array}$ & $\begin{array}{l}\text { Start codon/ } \\
\text { Stop codon }\end{array}$ & $\begin{array}{l}\text { PolyA- } \\
\text { signal }\end{array}$ & $\begin{array}{l}\text { Frameshift } \\
\text { mutation }\end{array}$ & $\begin{array}{l}\text { Premature } \\
\text { stop codon }\end{array}$ & $\begin{array}{l}\text { Longest ORF }=\text { RPSA (position) } \\
\text { identities/positives }\end{array}$ \\
\hline RPSAP1 & GQ202530 & $93 \%$ & ATG/TAA & Yes & No & Yes & $\begin{array}{l}83 \text { aa }(1-83) \\
95 \% / 95 \%\end{array}$ \\
\hline RPSAP2 & GQ202531 & $74 \%$ & ATG/TAA & Yes & Yes & Yes & $\begin{array}{l}70 \text { aа }(34-103) \\
56 \% / 63 \%\end{array}$ \\
\hline RPSAP3 & GQ202532 & $80 \%$ & ATG/TAA & No & Yes & Yes & $\begin{array}{l}84 \text { aa }(1-84) \\
82 \% / 87 \%\end{array}$ \\
\hline RPSAP4 & GQ202533 & $83 \%$ & No/No & No & Yes & N/A & $\begin{array}{l}90 \text { аa (10-99) } \\
48 \% / 53 \%\end{array}$ \\
\hline RPSAP5 & GQ202534 & $90 \%$ & ATG/TAA & Yes & Yes & Yes & $\begin{array}{l}129 \text { aa (10-138) } \\
91 \% / 92 \%\end{array}$ \\
\hline RPSAP6 & GQ202535 & $80 \%$ & $\mathrm{No} / \mathrm{No}$ & No & Yes & N/A & $\begin{array}{l}106 \text { aa }(160-265) \\
65 \% / 70 \%\end{array}$ \\
\hline RPSAP7 & GQ202536 & $99 \%$ & ATG/TAA & Yes & No & No & $\begin{array}{l}295 \text { aa }(1-295) \\
99 \% / 99 \%\end{array}$ \\
\hline RPSAPB & GQ202537 & $86 \%$ & $\mathrm{No} / \mathrm{TAA}$ & Yes & Yes & N/A & $\begin{array}{l}82 \text { aa }(177-258) \\
80 \% / 80 \%\end{array}$ \\
\hline RPSAP9 & GQ202538 & $98 \%$ & ATG/No & No & No & No & $\begin{array}{l}171 \text { aa }(1-171) \\
100 \% / 100 \%\end{array}$ \\
\hline RPSAP10 & GQ202539 & $94 \%$ & $\mathrm{No} / \mathrm{TAA}$ & Yes & Yes & N/A & $\begin{array}{l}57 \text { aа }(174-230) \\
95 \% / 100 \%\end{array}$ \\
\hline RPSAP11 & GQ202540 & $76 \%$ & No/No & Yes & Yes & N/A & $\begin{array}{l}107 \text { aa }(185-291) \\
46 \% / 49 \%\end{array}$ \\
\hline
\end{tabular}

Table 3 Characteristics of RPSA (pseudo)genes-repeats and transcription

\begin{tabular}{|c|c|c|c|}
\hline Gene & $\begin{array}{l}\text { Interspersed repeats in } \\
\text { gene }\end{array}$ & Flanking repeats/family/class & Transcription \\
\hline RPSAP1 & / & / & / \\
\hline RPSAP2 & / & 3': L2C/L2/LINE & $\begin{array}{l}\text { Cerebrum, cerebellum, spleen, muscle, lymph node and } \\
\text { duodenum }\end{array}$ \\
\hline RPSAP3 & / & $\begin{array}{l}\text { 5': ERV3-16A3_l-int/ERVL/LTR } \\
\text { 3': CHR-2A/tRNA-Glu/SINE }\end{array}$ & Cerebrum \\
\hline RPSAP4 & ART2A/RTE-BovB/SINE & $\begin{array}{l}\text { 5': L1M3/L1/LINE } \\
\text { 3': L1M3/L1/LINE and (CA)n/Simple_repeat and } \\
\text { L1M3/L1/LINE }\end{array}$ & Cerebrum, cerebellum and spleen \\
\hline RPSAP5 & / & l & Cerebrum \\
\hline RPSAP6 & ART2A/RTE-BovB/SINE & 3': CHRL/tRNA-Glu/SINEand tRNA-Glu-GAA/tRNA & I \\
\hline RPSAP7 & Bov-tA1/BovA/SINE & $\begin{array}{l}\text { 5': LTR16A2/ERVL/LTR } \\
\text { 3': L1MEC/L1/LINE and L1M3/L1/LINE }\end{array}$ & / \\
\hline RPSAP8 & I & / & Cerebrum and cerebellum \\
\hline RPSAP9 & / & $\begin{array}{l}\text { 5': MIR/SINE and MIR/SINE and L1M2/L1/LINE } \\
\text { 3': L1M2/L1/LINE and (CATA)n/Simple_repeat }\end{array}$ & / \\
\hline RPSAP10 & I & l & I \\
\hline RPSAP11 & / & $\begin{array}{l}\text { 5': Bov-tA2/BovA/SINE } \\
\text { 3': L1M5/L1/LINE }\end{array}$ & $\begin{array}{l}\text { Cerebrum, cerebellum, spleen, muscle, lymph node and } \\
\text { duodenum }\end{array}$ \\
\hline
\end{tabular}

RPSAP5 in EPHA6; Table 4), which confirms the fact that most processed pseudogenes persist in regions where they do not cause deleterious effects [22].

The genomic region around the ovine RPSA family members show conserved synteny (same genes, same orientation and same order) with the bovine genome. $L O C 784055$, probably a processed pseudogene of GOLPH3L located in intron 2 of GON4L on Bos taurus chromosome (BTA) 3 and expected in the ovine minicontig containing RPSAP2, was the only bovine ortholog not present in sheep and therefore is most probably a bovine specific pseudogene.

The flanking sequences (500 bp upstream and $500 \mathrm{bp}$ downstream) of each RPSA pseudogene were blasted against the bovine and human genome. Out of the 11 identified orthologous bovine sequences, 5 were 


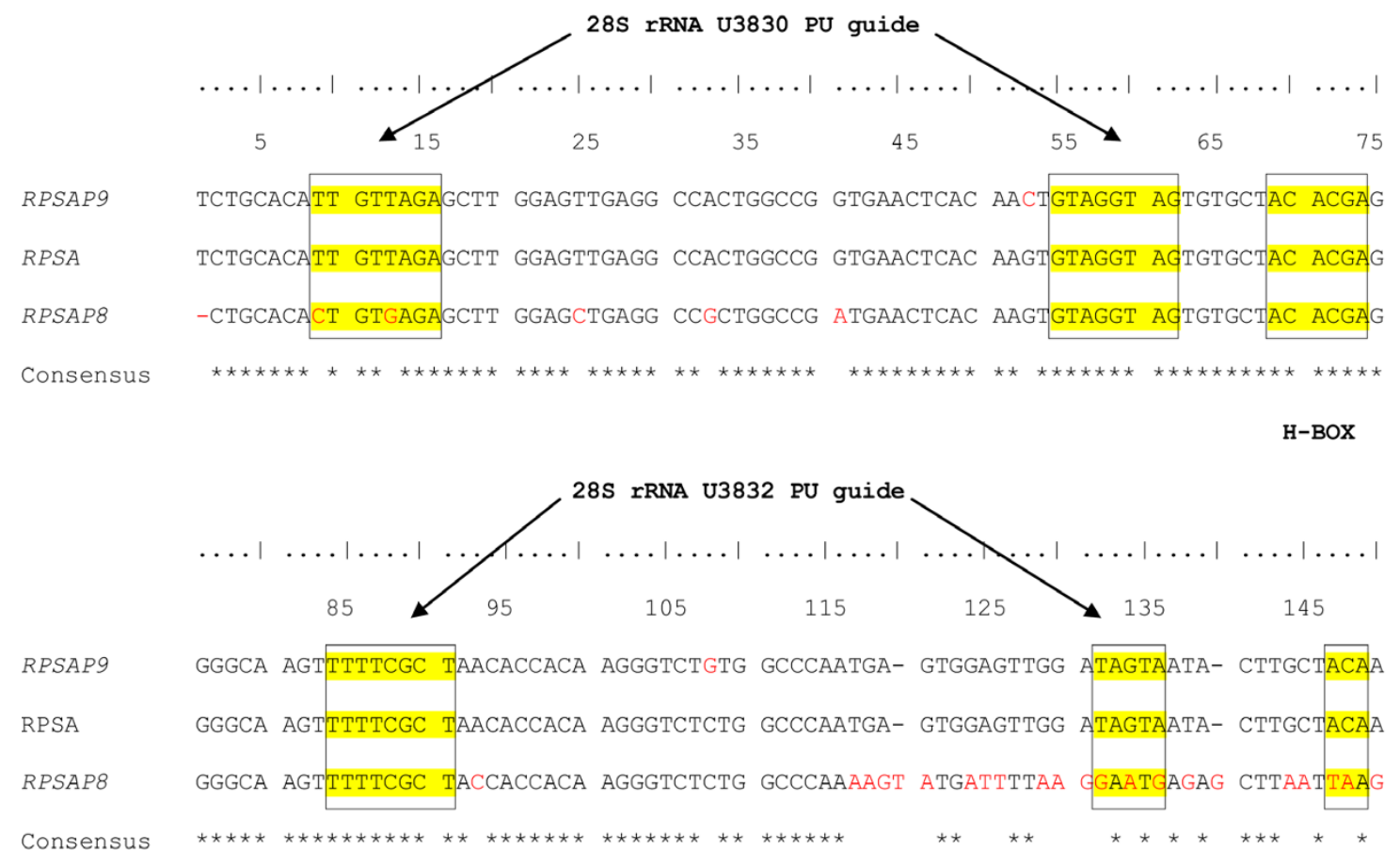

ACA-BOX

Figure 4 Alignment of the snoRNAs in RPSA, RPSAP8 and RPSAP9. The ACA-box, H-box and 285 rRNA U3830 and U3832 PU guide are highlighted in yellow.

interrupted by a bovine RPSA pseudogene; in the 6 other cases, the upstream sequence continued into the downstream sequence without an interruption of a pseudogene. The latter was also the case with the 11 orthologous human sequences. Thus we found 5 orthologous bovine RPSA pseudogenes but no human orthologs (Table 5).

A BLAST analysis of the bovine genome (reference assembly, based on Btau_4.0) with both ovine and bovine RPSA and RPSA pseudogene sequences identified 60 potential RPSA family members (Additional file 8). These included the only bovine pseudogene described so far, designated as RPSAP1 and located on BTA4 [32]. No ortholog of this pseudogene was found in sheep. Twenty-five sequences were annotated as 'similar to Ribosomal protein SA pseudogene' but only one corresponded to an ovine ortholog i.e. RPSAP11. To date, the 35 remaining sequences have not been annotated, but we have identified an ovine ortholog in 4 cases (Table 4 and 5; Additional file 8). Apart from RPSAP3, the ORF of the ovine and bovine orthologs differ substantially, suggesting that there is no selective pressure to conserve the ORF of these pseudogenes.

No bovine ortholog was found for the 6 sheep RPSA pseudogenes sharing 86 to $99 \%$ nucleotide identity with
RPSA whereas the 5, for which a bovine ortholog was identified, only displayed 74-83\% sequence identity with RPSA. As the amount of mutations accumulated by the pseudogenes during evolution can be used to infer their age [27], it's not surprising that the first group, consisting of recently arisen pseudogenes which have not yet accumulated many mutations, is lineage specific and that the pseudogenes of the latter group, comprising the oldest pseudogenes, all have a bovine ortholog. In addition, none of the 11 ovine pseudogenes were orthologous with any of the 63 annotated human RPSA pseudogenes. As a result, we can conclude that all 11 ovine RPSA pseudogenes detected originated after the divergence between primates and ungulates and 6 of these after the divergence between cattle and sheep.

\section{Transcription profiling by RT-PCR}

To investigate whether some of the ovine RPSA pseudogenes were potentially functional, transcription profiling was performed by RT-PCR for all sheep RPSA family members in 7 tissues (Figure 5) i.e. cerebrum, cerebellum, spleen, muscle, lymph node, duodenum and blood. To be sure that no genomic DNA was present in the RNA samples, they were treated with DNase and checked by minus RT control PCR (Additional file 9). 
Table 4 Location of RPSA (pseudo)genes

\begin{tabular}{lll}
\hline Gene & Chromosomal location & Ortholog Bos taurus \\
\hline RPSA & OAR19q13 intergenic between LOC515736 and MOBP & ortholog BTA22: GenelD: 281898 \\
\hline RPSAP1 & OAR20q22 intergenic between LOC401242 and LOC538046 & no \\
\hline RPSAP2 & OAR1p13 in intron 2 DAP3 & ortholog BTA3: not annotated yet \\
\hline RPSAP3 & OAR 5q22.3 intergenic between PCDHB15 and TAF7 & ortholog BTA7: not annotated yet \\
\hline RPSAP4 & OAR19q12 in intron 1 LOC789684 & ortholog BTA22: not annotated yet \\
\hline RPSAP5 & OAR1q21-q22 in intron 2 EPHA6 & no \\
\hline RPSAP6 & OAR17q26prox intergenic between PXN and SIRT4 & ortholog BTA17: not annotated yet \\
\hline RPSAP7 & OAR23q23prox intergenic between RBBP8 and LOC100138286 & no \\
\hline RPSAP8 & OAR7q12-q13 intergenic between SERINC5 and GNPNAT1 & no \\
\hline RPSAP9 & OAR1p37 intergenic between LOC100141009 and LOC522241 & no \\
\hline RPSAP10 & OAR17q21 prox intergenic between MDK and LOC783956 & no \\
\hline RPSAP11 & OAR23q21 intergenic between ZNF24 and LOC767868 & ortholog BTA24: GenelD: 100138378 \\
\hline
\end{tabular}

Table 5 Bovine orthologs

\begin{tabular}{|c|c|c|c|c|c|c|}
\hline $\begin{array}{l}\text { Ovine } \\
\text { ortholog }\end{array}$ & $\begin{array}{l}\text { Chromosomal } \\
\text { location }\end{array}$ & $\begin{array}{l}\text { GenBank } \\
\text { Acc. No. }\end{array}$ & Range & $\begin{array}{c}\text { Nucleic acid identity with } \\
\text { bovine } R P S A\end{array}$ & $\begin{array}{l}\text { Nucleic acid identity with } \\
\text { ovine ortholog }\end{array}$ & Features in sequence \\
\hline RPSA & BTA22 & NC_007320.3 & $\begin{array}{l}12885045- \\
12898467\end{array}$ & $100 \%$ & $\begin{array}{l}\text { CDS } 96 \% \\
\text { Gene } 87 \%\end{array}$ & $\begin{array}{l}\text { RPSA: } 12886519- \\
12898467\end{array}$ \\
\hline RPSAP2 & BTA3 & NC_007301.3 & $\begin{array}{l}16411109- \\
16410143\end{array}$ & $75 \%$ & $91 \%$ & $\begin{array}{l}\text { DAP3 intron 2: } \\
16430465-16408264\end{array}$ \\
\hline RPSAP3 & BTA7 & NC_007305.3 & $\begin{array}{l}51802201- \\
51801057\end{array}$ & $81 \%$ & $95 \%$ & $\begin{array}{l}\text { LOC786980: } 51801077- \\
51802181\end{array}$ \\
\hline RPSAP4 & BTA22 & NC_007320.3 & $\begin{array}{l}2925069- \\
2925867\end{array}$ & $78 \%$ & $91 \%$ & $\begin{array}{l}\text { LOC789684: } 2840530- \\
3050478\end{array}$ \\
\hline RPSAP6 & BTA17 & NC_007315.3 & $\begin{array}{l}65883767- \\
65884909\end{array}$ & $81 \%$ & $92 \%$ & $\begin{array}{l}\text { LOC783583: 65881027- } \\
65884941\end{array}$ \\
\hline RPSAP11 & BTA24 & NC_007325.3 & $\begin{array}{l}22831525- \\
22830341\end{array}$ & $77 \%$ & $91 \%$ & $\begin{array}{l}\text { LOC100138378: } \\
\text { 22830405-22834810 }\end{array}$ \\
\hline
\end{tabular}

For 8 members of the RPSA family, gene-specific primers could be designed and their specificity was proven by checking that the primers did not amplify any other RPSA family member using the respective unique BAC clones as template (Additional file 10). Because RPSA, RPSAP1, RPSAP7 and RPSAP9 share a high level of sequence identity, no specific primers could be designed for these RPSA family members, they were tested with aspecific primers. All generated amplicons were sequenced. RPSA was expressed in all tested tissues. This agrees with the results of Marcos-Carcavilla et al. and Qiao et al. $[18,33]$. None of the pseudogenes was transcribed in blood. RPSAP2 and RPSAP11 were transcribed in all other tested tissues, while RPSAP3, RPSAP5 and RPSAP8 were only transcribed in one or more brain regions and RPSAP4 was transcribed in brain regions and spleen. RPSAP6 and RPSAP10 were not expressed in any of the tested tissues. In the case of RPSAP1, RPSAP7 and RPSAP9, tested with aspecific primers which all could also amplify RPSA, we generated amplicons which, after sequencing, turned out to be all RPSA transcripts. Thus we can conclude that RPSAP1, RPSAP7 and RPSAP9 are not expressed or at a very low level compared to the active RPSA gene. Therefore it would be interesting to do RT-qPCR with specific probes in order to be sure if the pseudogenes are expressed at very low levels or not at all. No clear relationship between the transcription profile of the various pseudogenes and the in silico prediction of possible promoters was observed. For instance, RPSAP10 is not expressed in any tissue tested although we did predict a promoter in the upstream sequence. Thus it may be possible that RPSAP10 is expressed in other tissues not examined in this study or that it has a low level of transcription. In addition, the in silico predicted promoter might not act as a cis-regulatory element in vivo. In contrast, RPSAP3 is transcribed in certain brain regions although we did not predict any promoter, probably because the promoter is located more upstream than the region analyzed here. 


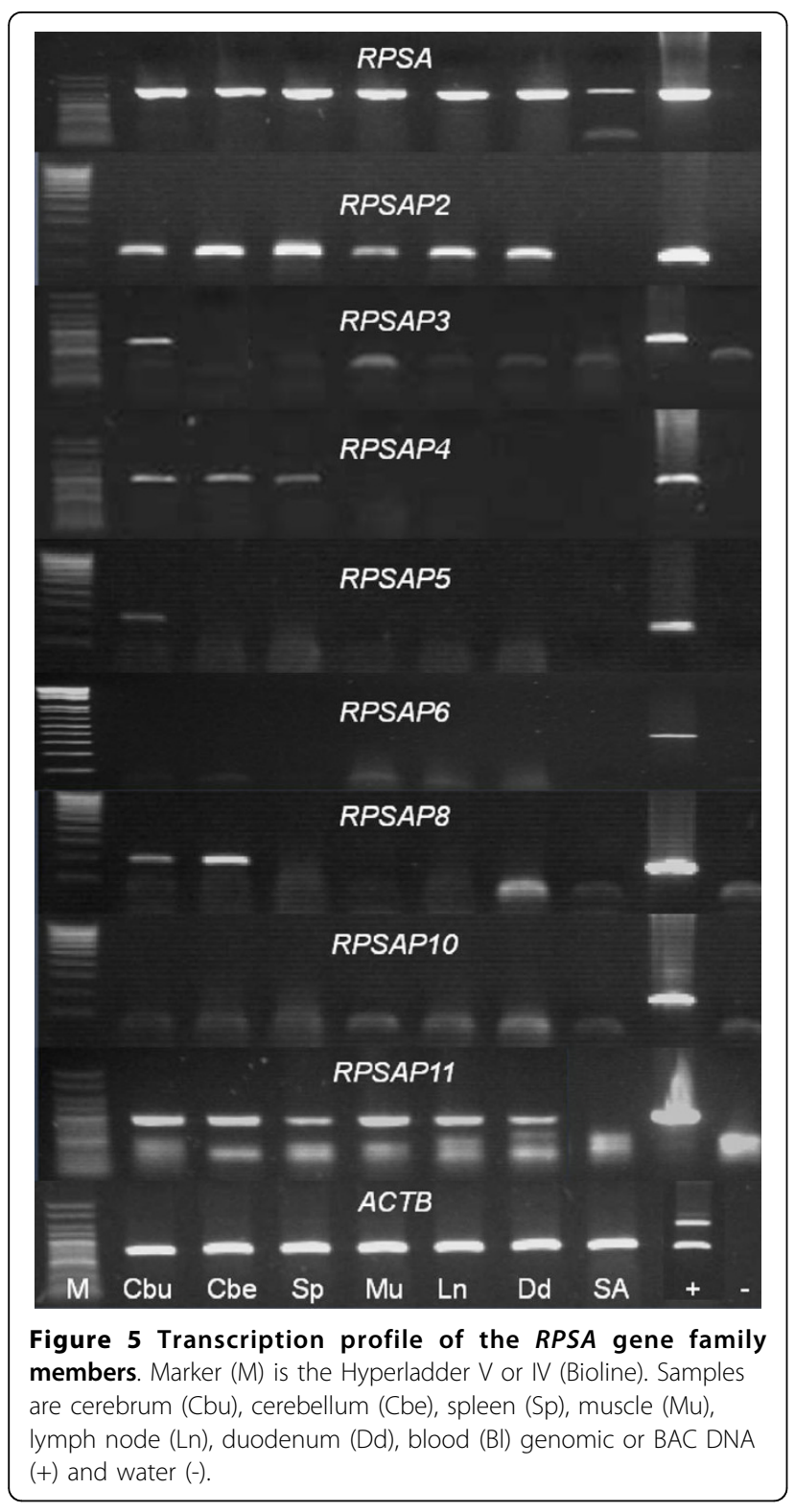

\section{Conclusions}

In addition to the already described ovine RPSA gene, we have identified 11 members of the ovine RPSA gene family, and designated them RPSAP1-RPSAP11 since they are all considered to be processed pseudogenes. The flanking genomic regions of each RPSA family member was analyzed by annotating the constructed BAC contigs, which revealed 40 genes (of which 37 had not been previously described in sheep) based on comparative mapping. All these regions show conserved synteny with the orthologous bovine counterparts and the locations were confirmed by FISH. Five pseudogenes have a bovine counterpart. In silico analysis predicted the presence of 55 more RPSA pseudogenes in the bovine genome.
Compared to the RPSA transcript, RPSA pseudogenes differ significantly both in structure and sequence identity, ranging from structurally identical pseudogenes sharing $99 \%$ sequence identity to pseudogenes lacking half of the gene or containing many deletions throughout the whole gene, sharing only $74 \%$ sequence identity. A remarkable result is that at least 6 of the 11 pseudogenes are transcriptionally active. However, whether these transcripts are functional as regulatory noncoding RNA or as functional proteins remains to be investigated.

In previous studies, 1 to 3 RPSA pseudogenes per species, discovered while screening with the intention to isolate the full length functional RPSA gene, were characterized. Furthermore, the number of RPSA pseudogenes in 4 species with fully sequenced genomes was determined by genome-wide in silico screening but those pseudogenes were not characterized (Table 1). Here we report in detail the characterization of the RPSA gene family in a species. A strategy was developed to isolate all the ovine RPSA pseudogenes sharing a high level of sequence identity with RPSA. We screened with 8 different primers representing each exon at least once and with a Ta that was at least $8^{\circ} \mathrm{C}$ lower than the Tm. All BAC clones were positive for at least 2 primer pairs and there was no concordance between the number of BAC in a mini-contig and the level of sequence identity with RPSA. Therefore, we conclude that it is most likely that we have isolated all the ovine RPSA pseudogenes that could interfere with the functional RPSA gene in genetic studies. The discrepancy between the numbers of ovine RPSA pseudogenes found (11) and the numbers described in genome-wide screenings (45-61) might be explained by the low sequence identity of most pseudogenes found in silico. In Bos taurus for instance, 51 of the 60 pseudogenes share an overall nucleic acid identity with the bovine RPSA gene beneath $80 \%$ (Additional file 8). Due to our experimental design, pseudogenes with a low sequence identity were not isolated since it is not likely that those pseudogenes would interfere with molecular studies on the functional full length RPSA gene.

In conclusion, we describe 11 ovine processed RPSA pseudogenes. This knowledge on their structure and sequence will facilitate the molecular genetic studies of the functional gene since it will now be possible to take into account the existence of the pseudogenes in the design of such studies.

\section{Methods}

\section{Construction BAC mini-contigs}

The ovine INRA BAC library, consisting of 90.000 clones with an average insert length of $123 \mathrm{~kb}$ and a genome equivalent of 3.4, was screened by PCR [19]. The primers were designed using Primer3, based on 
conserved regions in the sheep RPSA gene [34]. The conserved regions were detected by comparison of all ovine ESTs available in GenBank that shared similarity with the published ovine mRNA sequence of RPSA [GenBank:EF649775] with BLAST and ClustalW [21,35]. PCR was conducted with Faststart Taq DNA Polymerase (Roche). PCR conditions were $5 \mathrm{~min}$ at $95^{\circ} \mathrm{C}, 40$ cycles of $30 \mathrm{~s}$ at $95^{\circ} \mathrm{C}, 30 \mathrm{~s}$ at $50^{\circ} \mathrm{C}$ and $1 \mathrm{~min}$ at $72^{\circ} \mathrm{C}$, and a final 10-min elongation step at $72^{\circ} \mathrm{C}$. Thirty-nine superpools, each consisting of 44 pools (24 plates, 8 rows and 12 columns), were screened. Each positive combination was verified by colony PCR.

All isolated BACs were grown in a $200 \mathrm{ml}$ culture from which DNA was purified with the Qiagen Plasmid Midi kit (Qiagen) according to the manufacturer's instructions. The BAC ends were sequenced with the universal primer (UP) (5'-CGACGTTGTAAAACGACGGCCAG-3') and reverse primer (RP) (5'-CACAGGAAACAGCTATGACCATGATTACG-3') primers with $1 \mu \mathrm{g}$ of purified BAC DNA as template. Unique STS primer pairs (Additional file 2), based on the BESs, were used to screen all isolated BACs and to construct mini-contigs.

All sequencing was performed with the Big Dye Terminator mix (Applied Biosystems) and analyzed on an ABI-3730xl Analyser (Applied Biosystems).

\section{Characterization of RPSA gene family members}

The primers used to screen the INRA BAC library were used as initial sequence primers to sequence the RPSA family member in one BAC of each mini-contig by direct sequencing. The obtained sequence was then used to develop new sequencing primers until the whole gene and an additional $\pm 500 \mathrm{bp}$ upstream and $\pm 500 \mathrm{bp}$ downstream of the sequence showing similarity with RPSA, was sequenced. If the screening primer did not work as sequencing primer, the amplicon generated with the screening primer was cloned into a pCR 2.1 vector with the TA Cloning Kit (Invitrogen) and the vector was transformed in DH5 $\alpha$ Competent Cells (Invitrogen). The insert was then sequenced with UP and RP primers. All sequences were assembled into continuous sequences with CAP3 and analyzed with FGENESH and NCBI ORF Finder [20,36,37]. Promoter sequences were searched with CISTER, Neural Network Promoter Prediction, FPROM and TFsearch [36,38-40].

Annotation of the mini-contigs by comparative mapping All mini-contigs were annotated by comparing the BESs against bovine and human genomic sequences with NCBI BLAST [21]. Internal sequences were also annotated by PCR with primers based on bovine sequences of genes that were expected to be present in the minicontig. All amplicons were verified and repeats were detected with Repeatmasker [26].

\section{FISH}

Fluorescent in situ hybridization was performed at INRA in Jouy-en-Josas (France). To prepare the probes, BAC DNA was extracted according to standard protocols and purified with the S.N.A.P. K1900-01 Miniprep kit (Invitrogen). DNA was then nick-translated with biotin-14-dATP (BioNick 18247-015 labeling system, Invitrogen) and mixed with $100 \times$ total sonicated herring sperm DNA and $100 \times$ total sonicated sheep DNA. Subsequently, it was precipitated with ethanol, slightly dried and resuspended in hybridization buffer.

For R-banded sheep chromosomes, embryo fibroblast cell cultures were synchronized with an excess of thymidine and treated with 5-bromo-2'-deoxyuridine during the second half of $S$ phase [41].

$\mathrm{FISH}$, signal detection and R-banding were performed as previously described [42]. Briefly, labeled probes were denatured at $100^{\circ} \mathrm{C}$ for $10 \mathrm{~min}$ and pre-hybridized at $37^{\circ}$ $\mathrm{C}$ for 30 to $60 \mathrm{~min}$ before hybridization to the chromosomes. Chromosome identification and band numbering followed the standard sheep ideogram reported in ISCNDB2000 [43].

\section{Transcription profiling}

Fresh tissue samples were obtained from a commercial sheep slaughterhouse, frozen in liquid nitrogen immediately after slaughtering, crushed into powder and frozen at $-80^{\circ} \mathrm{C}$. Total RNA was isolated with the Aurum Total RNA Fatty and Fibrous Tissue kit (Bio-Rad) as described in the instruction manual. Subsequently, a minus RT-PCR was performed with actin, beta $(A C T B)$ primers on $1 \mu$ l RNA to confirm the absence of any DNA contamination (Additional file 9) as previously described [44]. If DNA was still present in the sample, an additional DNase treatment with RQ1 RNase-free DNase (Promega) and a spin column purification with Microcon YM-100 (Millipore) were carried out.

The RNA concentration and purity of the samples were measured with the Nanodrop ND-1000 Spectrophotometer (Isogen) and the RNA quality was determined by evaluation of the $28 \mathrm{~S}$ and $18 \mathrm{~S}$ ribosomal bands on a $0.8 \%$ agarose gel.

Then, 0.2-1 $\mu$ g RNA was converted into cDNA with iScript cDNA synthesis kit (Bio-Rad) using random and oligo dT primers. A confirmation PCR on $10 \times$ diluted cDNA with $A C T B$ primers (giving a different amplicon length on gDNA and cDNA) was performed.

Specific primers, based on the aligned sequences of the different RPSA family members (Additional file 3) were designed for 8 members of the RPSA family and specificity was proven (see above).

Due to the high level of nucleotide sequence identity among RPSA, RPSAP1, RPSAP7 and RPSAP9, it was not possible to develop specific primers for these, but we 
were able to develop several primer pairs which amplified different combinations of 2 to 6 RPSA family members. One primer for instance amplifies RPSA, RPSAP1, RPSAP7 and RPSAP11; another RPSA and RPSAP7 and a third one RPSA, RPSAP1, RPSAP5 RPSAP7 and RPSAP11.

The obtained amplicons were sequenced to determine/confirm which (pseudo)gene was transcribed.

\section{Additional file 1: Primers used for screening of the INRA BAC}

library. Characteristics of primers used for screening of the INRA BAC library: sequence, location, melting temperature and amplicon length Click here for file

[ http://www.biomedcentral.com/content/supplementary/1471-2164-11179-S1.XLSX]

Additional file 2: STS primers BES. Characteristics of primers used for STS content mapping: sequence, annealing temperature and amplicon length.

Click here for file

[http://www.biomedcentral.com/content/supplementary/1471-2164-11179-S2.XLSX]

Additional file 3: Alignment of the mRNA of the ovine RPSA gene with 11 RPSA pseudogenes. The startcodon, stopcodon, polyadenylation signal exon-exon junctions and interspersed repeats are highlighted in yellow. The primers used to screen the INRA BAC library are highlighted in green.

Click here for file

[http://www.biomedcentral.com/content/supplementary/1471-2164-11 179-S3.PDF ]

Additional file 4: In silico promoter prediction. The in silico predicted promoter sequence is shown with the transcription start site highlighted in red. Furthermore, the score of the prediction, identification of the promoter and location of the transcription start site in the sequence is shown.

Click here for file

[http://www.biomedcentral.com/content/supplementary/1471-2164-11179-S4.XLSX]

Additional file 5: Characteristics BES. Characteristics of the 68 BES: BES length, orthologous sequences and genes, and repeats present in the BES.

Click here for file

[http://www.biomedcentral.com/content/supplementary/1471-2164-11179-S5.XLSX]

Additional file 6: Primers used to annotate the mini-contigs. Characteristics of primers used to annotate the mini-contig: sequence, annealing temperature, amplicon size and annotation information. Click here for file

[http://www.biomedcentral.com/content/supplementary/1471-2164-11179-S6.XLSX]

Additional file 7: FISH experiments. Pictures of FISH experiments of the 11 RPSA pseudogenes.

Click here for file

[http://www.biomedcentral.com/content/supplementary/1471-2164-11179-S7.PDF ]

Additional file 8: Bovine RPSA pseudogenes. Characteristics of bovine RPSA pseudogenes predicted in silico by BLAST analysis of the bovine genome (reference assembly, based on Btau_4.0). In yellow are the pseudogenes that are annotated as 'similar to Ribosomal protein SA pseudogene'. The bovine pseudogene RPSAP1 is highlighted in blue and the pseudogenes that aren't annotated yet in white.

Click here for file

[http://www.biomedcentral.com/content/supplementary/1471-2164-11179-S8.XLSX]
Additional file 9: minus-RT PCR control on RNA isolated from blood RT-PCR with ACTB primers. Marker (M) is the Hyperladder V (Bioline). Samples are RNA isolated from blood, genomic DNA and water (-).

Click here for file

[http://www.biomedcentral.com/content/supplementary/1471-2164-11179-S9.PDF ]

Additional file 10: Specificity of the expression primer for RPSAP6 PCR with expression primer for RPSAP6. Marker (M) is the Hyperladder IV (Bioline). Samples are the respective unique BAC clones of all RPSA family members, genomic DNA and water (-).

Click here for file

[http://www.biomedcentral.com/content/supplementary/1471-2164-11179-S10.PDF ]

\section{Acknowledgements}

The authors wish to thank Caroline Rogiers and Robin Maene for excellent technical assistance and Dr. Malena Serrano for her continuous help. Alice Van den Broeke is supported with a grant of the Institute for the Promotion of Innovation by Science and Technology in Flanders (IWT). Dr. MarcosCarcavilla was supported by RTA2006-00104 457 INIA project.

\section{Author details}

'Department of Nutrition, Genetics and Ethology, Faculty of Veterinary Medicine, Ghent University, Heidestraat 19, B-9820 Merelbeke, Belgium. ${ }^{2}$ Departamento de Mejora Genética Animal, INIA, Ctra La Coruña Km 7.5, Madrid 28040, Spain. ${ }^{3}$ INRA, UMR 1313 Génétique Animale et Biologie Intégrative, F78350 Jouy-en-Josas, France.

\section{Authors' contributions}

AVDB carried out the BAC library screening, mini-contig building, sequencing of the genes, annotation by comparative mapping, the transcription profiling and drafted this manuscript. MVP participated in the design of the study, participated in the screening of the BAC library and provided experimental support. AMC participated in the screening of the BAC library. KH supervised the BAC library screening. $\mathrm{HH}$ and MB carried out the FISH mapping experiments. AVZ supervised the study. LJP participated in the study design and supervised the study. All authors read and approved the final manuscript.

Received: 9 December 2009 Accepted: 16 March 2010

Published: 16 March 2010

\section{References}

1. Kinoshita K, Kaneda Y, Sato M, Saeki Y, Wataya-Kaneda M, Hoffmann A: LBPp40 binds DNA tightly through associations with histones $\mathrm{H} 2 \mathrm{~A}, \mathrm{H} 2 \mathrm{~B}$, and H4. Biochemical and biophysical research communications 1998, 253(2):277-282.

2. Nelson J, McFerran NV, Pivato G, Chambers E, Doherty C, Steele D, Timson DJ: The $67 \mathrm{kDa}$ laminin receptor: structure, function and role in disease. Bioscience reports 2008, 28(1):33-48.

3. Orihuela CJ, Mahdavi J, Thornton J, Mann B, Wooldridge KG, Abouseada N, Oldfield NJ, Self T, Ala'Aldeen DA, Tuomanen El: Laminin receptor initiates bacterial contact with the blood brain barrier in experimental meningitis models. J Clin Invest 2009, 119(6):1638-1646.

4. Chen FX, Qian YR, Duan YH, Ren WW, Yang Y, Zhang CC, Qiu YM, Ji YH: Down-regulation of $67 \mathrm{LR}$ reduces the migratory activity of human glioma cells in vitro. Brain Res Bull 2009, 79(6):402-408.

5. Zhou L, Xie M, Zhou JQ, Tao L: 67-kDa laminin receptor in human laryngeal squamous cell carcinoma. Laryngoscope 2006, 116(1):28-32.

6. Li D, Chen J, Gao Z, Li X, Yan X, Xiong Y, Wang S: 67-kDa laminin receptor in human bile duct carcinoma. Eur Surg Res 2009, 42(3):168-173.

7. Zuber C, Knackmuss S, Zemora G, Reusch U, Vlasova E, Diehl D, Mick V, Hoffmann K, Nikles D, Frohlich T, Arnold GJ, Brenig B, Wolf E, Lahm H, Little M, Weiss S: Invasion of tumorigenic HT1080 cells is impeded by 
blocking or downregulating the $37-\mathrm{kDa} / 67-\mathrm{kDa}$ laminin receptor. Journal of molecular biology 2008, 378(3):530-539.

8. Zuber C, Knackmuss S, Rey C, Reusch U, Rottgen P, Frohlich T, Arnold GJ, Pace C, Mitteregger G, Kretzschmar HA, Little M, Weiss S: Single chain Fv antibodies directed against the $37 \mathrm{kDa} / 67 \mathrm{kDa}$ laminin receptor as therapeutic tools in prion diseases. Molecular immunology 2008, 45(1):144-151.

9. Zuber C, Mitteregger G, Schuhmann N, Rey C, Knackmuss S, Rupprecht W, Reusch U, Pace C, Little M, Kretzschmar HA, Hallek M, Buning H, Weiss S: Delivery of single-chain antibodies (scFvs) directed against the 37/67 $\mathrm{kDa}$ laminin receptor into mice via recombinant adeno-associated viral vectors for prion disease gene therapy. The Journal of general virology 2008, 89(Pt 8):2055-2061.

10. Pflanz H, Vana K, Mitteregger G, Pace C, Messow D, Sedlaczek C, Nikles D, Kretzschmar HA, Weiss SF: Microinjection of lentiviral vectors expressing small interfering RNAs directed against laminin receptor precursor mRNA prolongs the pre-clinical phase in scrapie-infected mice. The Journal of general virology 2009, 90(Pt 1):269-274.

11. Zuber C, Mitteregger G, Pace C, Zerr I, Kretzschmar HA, Weiss S: Anti-LRP/ LR antibody W3 hampers peripheral PrPSC propagation in scrapie infected mice. Prion 2007, 1(3):207-212.

12. Pflanz H, Vana K, Mitteregger G, Renner-Muller I, Pace C, Kuchenhoff $H$, Kretzschmar HA, Wolf E, Weiss S: Scrapie-infected transgenic mice expressing a laminin receptor decoy mutant reveal a prolonged incubation time associated with low levels of PrPres. Journal of molecular biology 2009, 388(4):721-729.

13. Holy EW, Stampfli SF, Akhmedov A, Holm N, Camici GG, Luscher TF, Tanner FC: Laminin receptor activation inhibits endothelial tissue factor expression. J Mol Cell Cardiol 2009.

14. Fujimura Y, Umeda D, Yamada K, Tachibana H: The impact of the $67 \mathrm{kDa}$ laminin receptor on both cell-surface binding and anti-allergic action of tea catechins. Arch Biochem Biophys 2008, 476(2):133-138.

15. Ku HC, Chang HH, Liu HC, Hsiao CH, Lee MJ, Hu YJ, Hung PF, Liu CW, Kao YH: Green tea (-)-epigallocatechin gallate inhibits insulin stimulation of 3T3-L1 preadipocyte mitogenesis via the $67-\mathrm{kDa}$ laminin receptor pathway. Am J Physiol Cell Physiol 2009, 297(1):C121-132.

16. Umeda D, Yano S, Yamada K, Tachibana H: Green tea polyphenol epigallocatechin-3-gallate signaling pathway through $67-k \mathrm{Da}$ laminin receptor. The Journal of biological chemistry 2008, 283(6):3050-3058.

17. Khachane AN, Harrison PM: Assessing the genomic evidence for conserved transcribed pseudogenes under selection. BMC Genomics 2009, 10:435.

18. Marcos-Carcavilla A, Calvo JH, Gonzalez C, Serrano C, Moazami-Goudarzi K, Laurent P, Bertaud M, Hayes $H$, Beattie AE, Lyahyai J, Martin-Burriel I, Torres $J \mathrm{M}$, Serrano M: Structural and functional analysis of the ovine laminin receptor gene (RPSA): Possible involvement of the LRP/LR protein in scrapie response. Mamm Genome 2008, 19(2):92-105.

19. Vaiman D, Billault A, Tabet-Aoul K, Schibler L, Vilette D, Oustry-Vaiman A, Soravito C, Cribiu EP: Construction and characterization of a sheep BAC library of three genome equivalents. Mamm Genome 1999, 10(6):585-587.

20. Huang X, Madan A: CAP3: A DNA sequence assembly program. Genome research 1999, 9(9):868-877.

21. Altschul SF, Gish W, Miller W, Myers EW, Lipman DJ: Basic local alignment search tool. Journal of molecular biology 1990, 215(3):403-410.

22. Mighell AJ, Smith NR, Robinson PA, Markham AF: Vertebrate pseudogenes. FEBS Lett 2000, 468(2-3):2-3.

23. Zhang Z, Carriero N, Gerstein M: Comparative analysis of processed pseudogenes in the mouse and human genomes. Trends Genet 2004, 20(2):62-67.

24. Ding W, Lin L, Chen B, Dai J: L1 elements, processed pseudogenes and retrogenes in mammalian genomes. IUBMB Life 2006, 58(12):677-685

25. Hundt C, Peyrin JM, Haik S, Gauczynski S, Leucht C, Rieger R, Riley ML, Deslys JP, Dormont D, Lasmezas Cl, Weiss S: Identification of interaction domains of the prion protein with its $37-\mathrm{kDa} / 67-\mathrm{kDa}$ laminin receptor. The EMBO journal 2001, 20(21):5876-5886.

26. Repeatmasker Webserver. [http://www.repeatmasker.org].

27. Zhang Z, Harrison P, Gerstein M: Identification and analysis of over 2000 ribosomal protein pseudogenes in the human genome. Genome research 2002, 12(10):1466-1482.
28. Zhang ZD, Cayting P, Weinstock G, Gerstein M: Analysis of nuclear receptor pseudogenes in vertebrates: how the silent tell their stories. Molecular biology and evolution 2008, 25(1):131-143.

29. Zemann A, op de Bekke A, Kiefmann M, Brosius J, Schmitz J: Evolution of small nucleolar RNAs in nematodes. Nucleic acids research 2006 , 34(9):2676-2685.

30. Lestrade L, Weber MJ: snoRNA-LBME-db, a comprehensive database of human H/ACA and C/D box snoRNAs. Nucleic acids research 2006, 34 Database: D158-162.

31. Reichow SL, Hamma T, Ferre-D'Amare AR, Varani G: The structure and function of small nucleolar ribonucleoproteins. Nucleic acids research 2007, 35(5):1452-1464.

32. Germerodt M, Knorr C, Beck J, Drogemuller C, Williams JL, Habermann F, Fries R, Brenig B: Characterization and chromosome localization of a processed pseudogene related to the bovine laminin receptor gene family. Cytogenet Genome Res 2004, 107(1-2):1-2.

33. Qiao J, Su X, Wang Y, Yang J, Kouadir M, Zhou X, Yin X, Zhao D: Cloning and characterization of full-length coding sequence (CDS) of the ovine 37/67-kDa laminin receptor (RPSA). Mol Biol Rep 2009, 36(8):2131-2137.

34. Rozen S, Skaletsky H: Primer3 on the WWW for general users and for biologist programmers. Methods Mol Biol 2000, 132:365-386.

35. ClustalW Webserver. [http://www.ebi.ac.uk/Tools/clustalw].

36. FGENESH Webserver. [http://linux1.softberry.com].

37. NCBI ORF Finder Webserver. [http://www.ncbi.nlm.nih.gov/projects/gorf/]

38. Cister Webserver. [http://zlab.bu.edu/ mfrith/cister.shtml].

39. Neural Network Promoter Prediction Webserver. [http://www.fruitfly.org/ seq_tools/promoter.html].

40. TFsearch Webserver. [http://www.cbrc.jp/research/db/TFSEARCH.html]

41. Hayes H, Petit E, Dutrillaux B: Comparison of RBG-banded karyotypes of cattle, sheep, and goats. Cytogenet Cell Genet 1991, 57(1):51-55.

42. Hayes $\mathrm{H}$, Petit E, Lemieux N, Dutrillaux B: Chromosomal localization of the ovine beta-casein gene by non-isotopic in situ hybridization and Rbanding. Cytogenet Cell Genet 1992, 61(4):286-288.

43. Cribiu EP, Di Berardino D, Di Meo GP, Eggen A, Gallagher DS, Gustavsson I, Hayes H, lannuzzi L, Popescu CP, Rubes J, Schmutz S, Stranzinger G, Vaiman A, Womack J: International System for Chromosome Nomenclature of Domestic Bovids (ISCNDB 2000). Cytogenet Cell Genet 2001, 92(3-4):3-4.

44. Lampo E, Van Poucke M, Hugot K, Hayes H, Van Zeveren A, Peelman L: Characterization of the genomic region containing the Shadow of Prion Protein (SPRN) gene in sheep. BMC Genomics 2007, 8:138.

45. Balasubramanian S, Zheng D, Liu YJ, Fang G, Frankish A, Carriero N, Robilotto R, Cayting P, Gerstein M: Comparative analysis of processed ribosomal protein pseudogenes in four mammalian genomes. Genome Biol 2009, 10(1):R2

46. Asano Y, Takashima S, Asakura M, Shintani Y, Liao Y, Minamino T, Asanuma H, Sanada S, Kim J, Ogai A, Fukushima T, Oikawa Y, Okazaki Y, Kaneda Y, Sato M, Miyazaki J, Kitamura S, Tomoike H, Kitakaze M, Hori M: Lamr1 functional retroposon causes right ventricular dysplasia in mice. Nat Genet 2004, 36(2):123-130.

47. Knorr C, Beuermann C, Beck J, Brenig B: Characterization of the porcine multicopy ribosomal protein $\mathrm{SA} / 37-\mathrm{kDa}$ laminin receptor gene family. Gene 2007, 395(1-2):1-2.

48. Fernandez MT, Castronovo V, Rao CN, Sobel ME: The high affinity murine laminin receptor is a member of a multicopy gene family. Biochemical and biophysical research communications 1991, 175(1):84-90.

49. Bignon C, Roux-Dosseto M, Zeigler ME, Mattei MG, Lissitzky JC, Wicha MS, Martin PM: Genomic analysis of the 67-kDa laminin receptor in normal and pathological tissues: circumstantial evidence for retroposon features. Genomics 1991, 10(2):481-485.

doi:10.1186/1471-2164-11-179

Cite this article as: Van den Broeke et al:: Characterization of the ovine ribosomal protein SA gene and its pseudogenes. BMC Genomics 2010 11:179. 